\title{
The need for holistic, longitudinal and comparable, real-time assessment of the emotional, behavioral and societal impact of the COVID-19 pandemic across nations
}

ARTICLE HISTORY: Received 5 March 2021/Revised 7 March 2021/Published Online 17 March 2021

As of the end of 2020, the COVID-19 pandemic has led to over 82 million verified infections and almost 1.8 million COVID-19related deaths worldwide, ${ }^{1}$ resulting to an unprecedented public health response around the globe. The COVID-19 pandemic, together with the applied multi-level restrictive measures, has generated a unique combination of an unpredictable and stressful biomedical and socioeconomic environment (i.e., syndemic), ${ }^{2}$ introducing real-life threat, involuntary and drastic every-day lifestyle changes with uncertain financial and future prospects, alongside with minimized coping and stress management possibilities. $^{3}$ This combination of so many different and vital stressors may lead to acute as well as long-term, direct, indirect and even transgenerational unfavourable effects on physical and mental health and functioning, which might even represent the most precarious and still unpredictable public-health-related part of the pandemic. ${ }^{4}$ Thereby, specific population groups could be at particular risk of poor health outcomes in relation to applied public health measures. ${ }^{4,5}$

However, not every individual will experience the same level of negative impact on health and well-being during the pandemic, as several additional national, socioeconomic, environmental, behavioural, emotional and cognitive factors can moderate individual resilience and coping. ${ }^{6}$ Pandemic-related research should, thus, assess as many multidimensional risk and protective factors as possible in a longitudinal, large-scale and multi-national manner, enabling a profound and comprehensive understanding of the complex health and societal impact of the pandemic worldwide. ${ }^{7}$

Nevertheless, to date, most research findings are cross-sectional, report on small and non-representative samples from individual countries, or on specific population groups (e.g., health care workers, students, clinical populations) and usually assess only a very restricted set of outcomes and time-points. Thereby, only few studies assess coping strategies, medical history or detailed socioeconomic, demographic and environmental data. In addition, most studies leave behind linguistic differences, being available in one or at best two different languages. Such investigations of small outcome subsets within a narrow framework preclude a broader and clear understanding of the multifaceted pandemic impact on the general population and specific subgroups. Acknowledging these gaps in the existing literature, large-scale, collaborative research prospectively collecting and monitoring a broad range of real-time, multi-dimensional health-related, societal and behavioural outcome data from countries across the globe is currently explicitly needed.

The Collaborative Outcomes study on Health and Functioning during Infection Times (COH-FIT) envisions to fill this gap. Based on an easy-to-access webpage (www.coh-fit.com), $\mathrm{COH}-\mathrm{FIT}$ is the currently largest-scale known international collaborative study of over 200 researchers around the globe, prospectively collecting the biggest set of multi-dimensional and multi-disciplinary data from 150 high, middle, and low-income countries in over 30 languages and in three different age groups (adults, adolescents, children) of the general population, focusing also on relevant at-risk subgroups. Albeit being a cross-sectional anonymous survey on an individual level, it is a longitudinal study on a population level, as data are collected continuously since April 2020 and until the WHO declares the end of the pandemic. In addition to snowball recruitment, this project also collects information from nationally representative samples. Furthermore, COH-FIT is the first study of this scale investigating pandemic effects on health and functioning measures between family members, while it also specifically assesses a large list of behavioral and coping factors (e.g., screen time, social media usage, physical activity, social interaction, religious practices, etc.) on outcomes of interest. $\mathrm{COH}-\mathrm{FIT}$ also monitors changes in public health restrictive measures to enhance data harmonization across nations and time, and to better investigate their impact on physical and mental health, while it also collects information on changes in healthcare systems functioning. The $\mathrm{COH}-\mathrm{FIT}$ project was worldwide first initiated in Greece after the ethics committee approval of the School of Medicine of the Aristotle University of Thessaloniki and is officially supported by the Hellenic Psychiatric Association, European Psychiatric Association, World Association of Social Psychiatry, ECNP Network on the Prevention of Mental Disorders and Mental Health Promotion, among many other national and international scientific associations. To date, $\mathrm{COH}-\mathrm{FIT}$ has already collected 
$>127,000$ participations worldwide (>8,900 in Greece), but more participants are still needed, both during the second and third wave of the pandemic, as in the future, after the pandemic has ended.

Currently, the COH-FIT survey actively collects the largest sample on multifactorial data on the impact of the COVD-19 pandemic on health and functioning not only in Greece, but around the globe. The elaborated design of COH-FIT and similar studies may allow a better identification of key parameters and population groups at increased risk during the pandemic, as well as potential targets for acute and long-term prevention or intervention strategies in the current as in possible future pandemics. A profound understanding of the health and societal impact of the pandemic could facilitate an optimized governmental, social and individual health preparedness during infection times ${ }^{8}$ and the bridging of individuals', societal and systemic needs and actions through multi-level guideline development with the aim to improve mental health outcomes globally.

\author{
Agorastos Agorastos \\ Assistant Professor of Psychiatry \\ 2nd Department of Psychiatry, School of Medicine, Aristotle University of Thessaloniki, Greece \\ Konstantinos Tsamakis \\ Research Associate \\ Institute of Psychiatry, Psychology and Neuroscience, King's College London, UK \\ Marco Solmi \\ Assistant Professor of Psychiatry \\ Department of Neuroscience, University of Padua, Italy \\ Christoph U. Correll \\ Professor of Child and Adolescent Psychiatry \\ Department of Child \& Adolescent Psychiatry, Psychotherapy and Psychosomatics, \\ Charité University Medical Center Berlin, Germany \\ Professor of Psychiatry and Molecular Medicine \\ Donald and Barbara Zucker School of Medicine at Hofstra/Northwell Hempstead, NY, USA \\ Vasilis P. Bozikas \\ Professor of Psychiatry \\ 2nd Department of Psychiatry, School of Medicine, Aristotle University of Thessaloniki, Greece
}

\section{References}

1. COVID-19 Dashboard Center for Systems Science and Engineering, Johns Hopkins University. Available from: https://www.arcgis.com/apps/opsdashboard/index.html\#/bda7594740fd40299423467b48e9ecf6. Accessed 31.12.2020

2. Horton R. Offline: COVID-19 is not a pandemic. Lancet 2020, 396:874, doi: 10.1016/S0140-6736(20)32000-6

3. Taylor S, Landry CA, Paluszek MM, Fergus TA, McKay D, Asmundson GJG. COVID stress syndrome: Concept, structure, and correlates. Depress Anxiety 2020, 37:706-714, doi: 10.1002/da.23071

4. Luo Y, Chua CR, Xiong Z, Ho RC, Ho CSH. A Systematic Review of the Impact of Viral Respiratory Epidemics on Mental Health: An Implication on the Coronavirus Disease 2019 Pandemic. Front Psychiatry 2020, 11:565098, doi: 10.3389/fpsyt.2020.565098
5. Salazar de Pablo G, Vaquerizo-Serrano J, Catalan A, Arango C, Moreno $C$, Ferre $F$ et al. Impact of coronavirus syndromes on physical and mental health of health care workers: Systematic review and meta-analysis. J Affect Disord 2020, 275:48-57, doi: 10.1016/j.jad. 2020.06.022

6. Kuntz JC. Resilience in times of global pandemic: Steering recovery and thriving trajectories. Appl Psychol 2020, Online ahead of print, doi: 10.1111/apps.12296

7. Tsamakis K, Dimitrakakis G, Stefanadi E, Tsiptsios DA, Dimitrakaki I, Mueller $\mathrm{C}$ et al (Comment) The challenges of planetary mental health in the COVID-19 era. Exp Ther Med 2020, 20:1843-1844, doi: 10.3892/etm. 2020.8927

8. Ahmad A, Mueller C, Tsamakis K. Covid-19 pandemic: a public and global mental health opportunity for social transformation? BMJ 2020, 369:1383, doi: 10.1136/bmj.m1383 


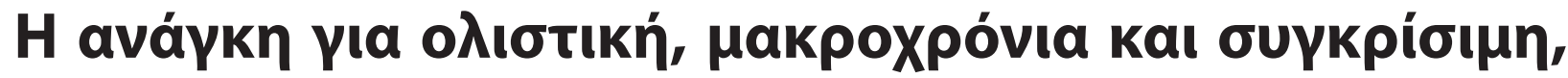

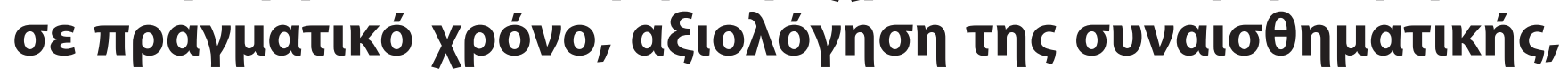

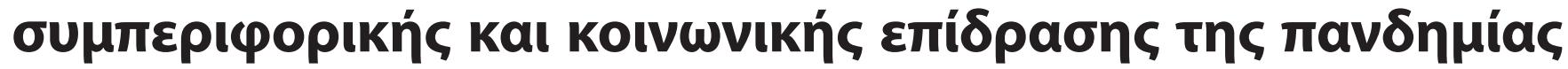

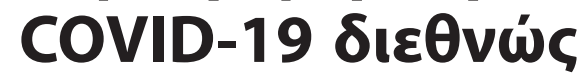

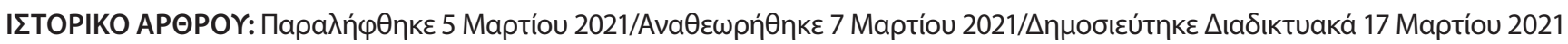

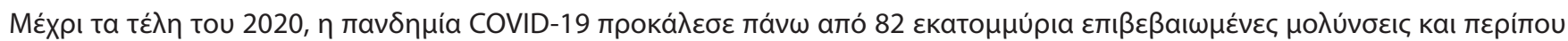

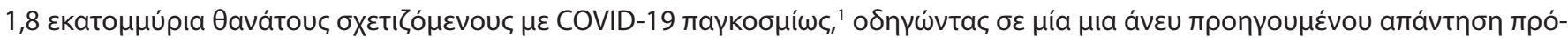

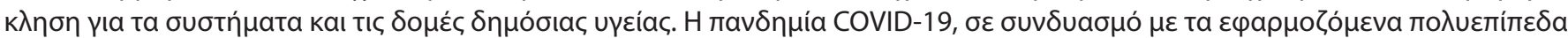

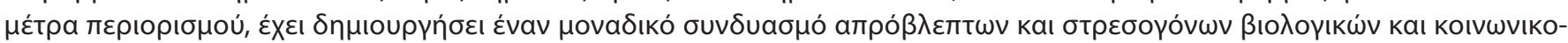

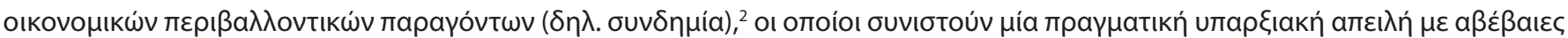

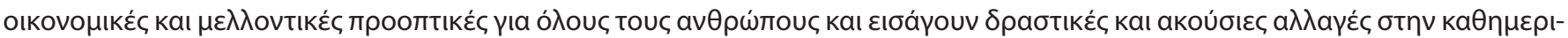

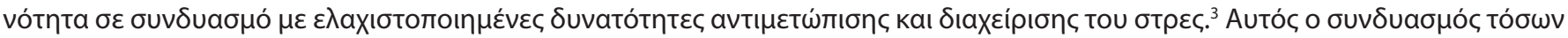

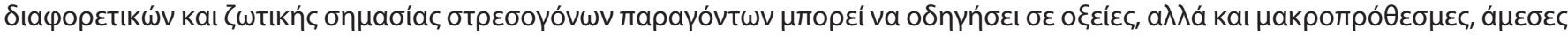

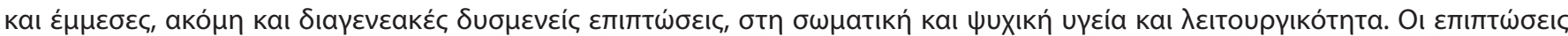

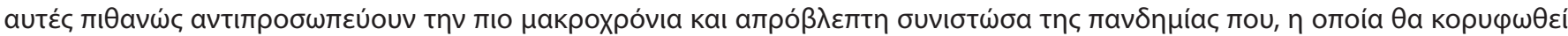

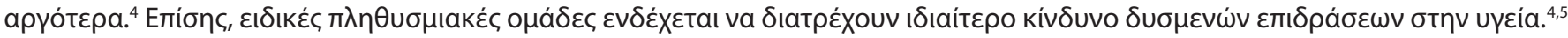

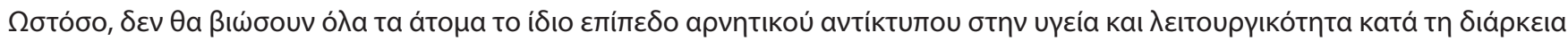

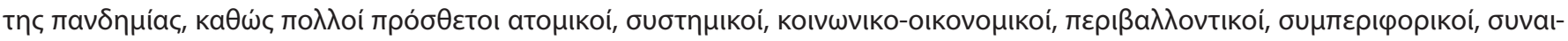

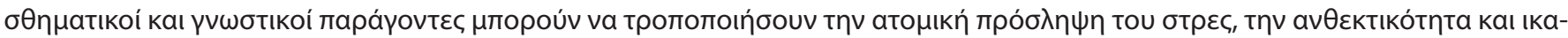

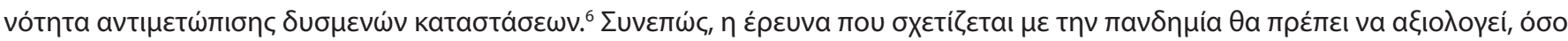

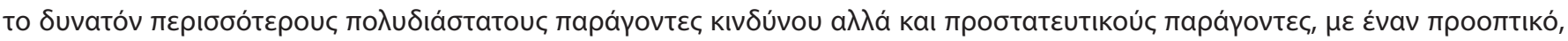

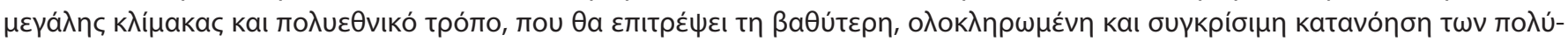

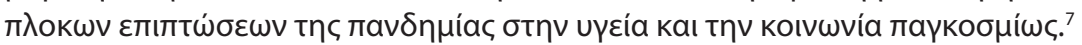

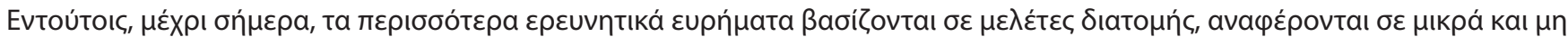

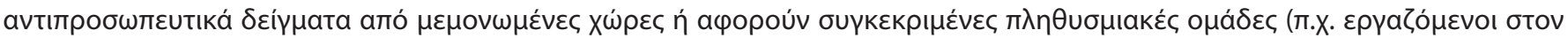

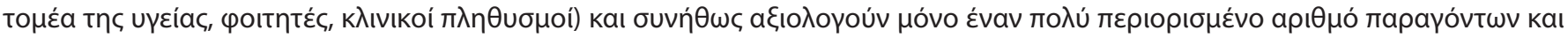

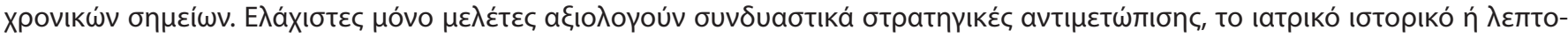

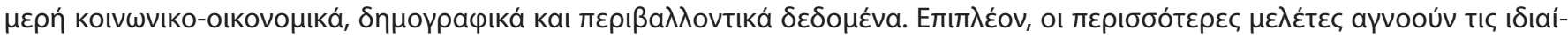

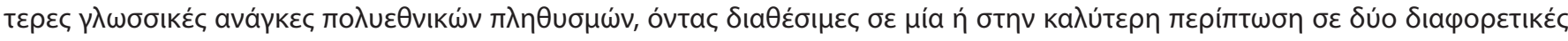

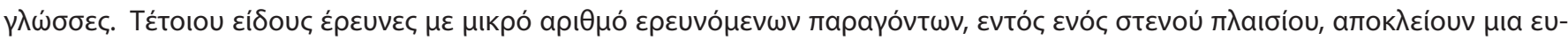

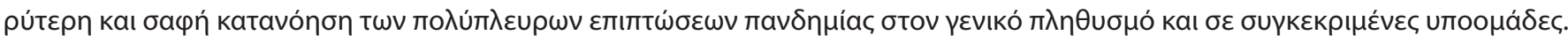

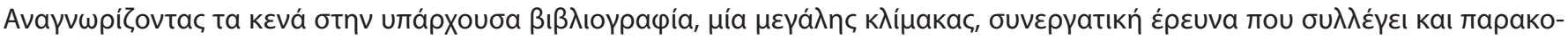

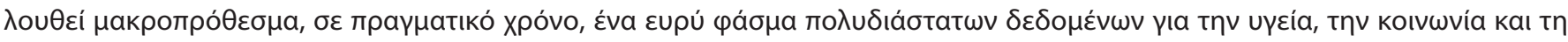

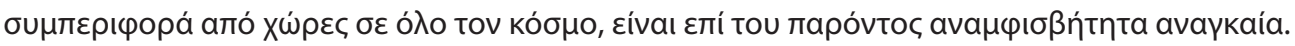

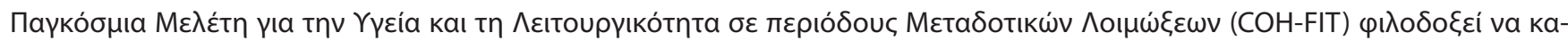

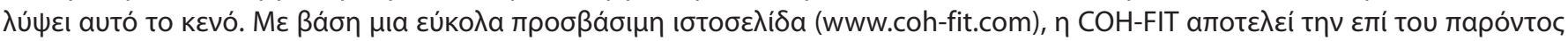

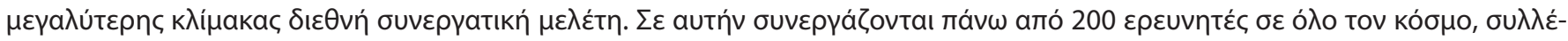

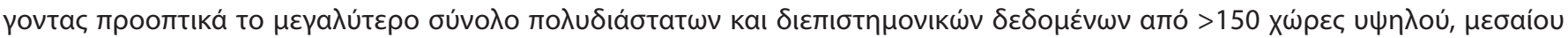

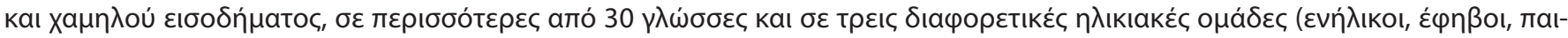

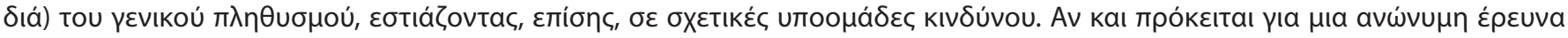

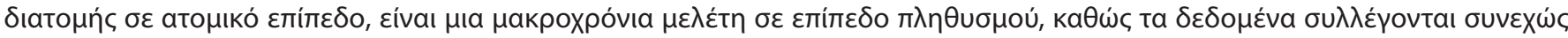

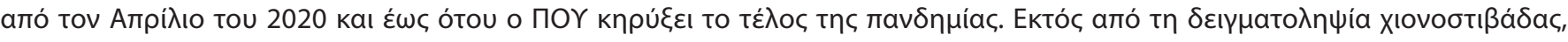




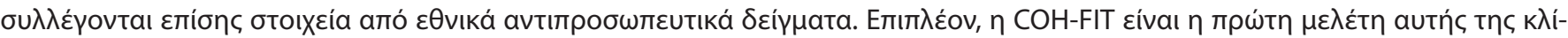

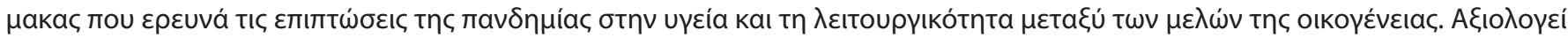

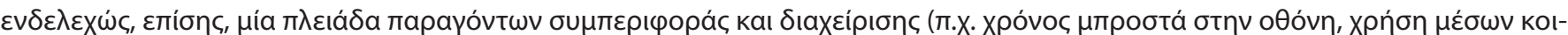

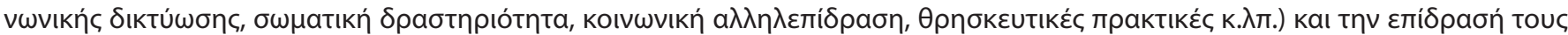

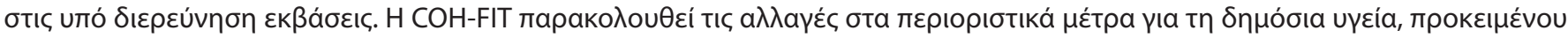

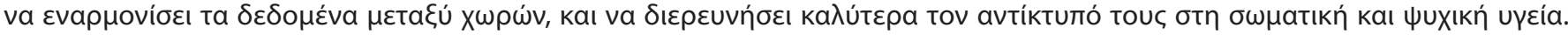

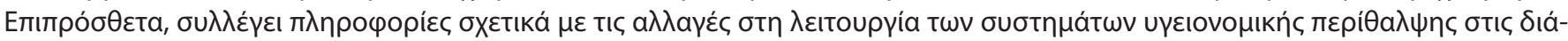

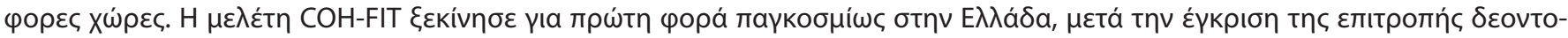

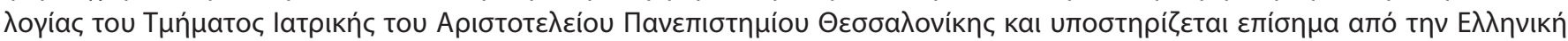

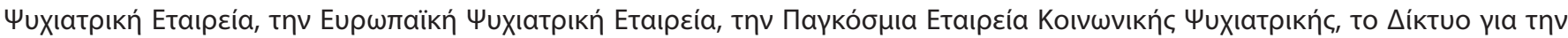

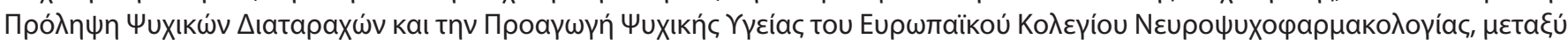

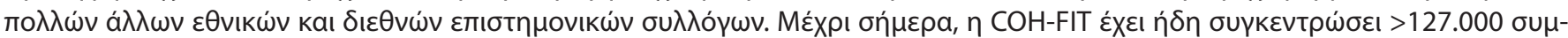
$\mu \varepsilon t \varepsilon ́ \chi$ X

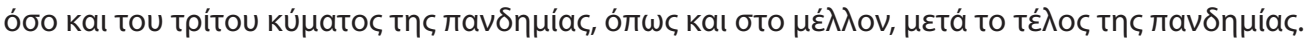

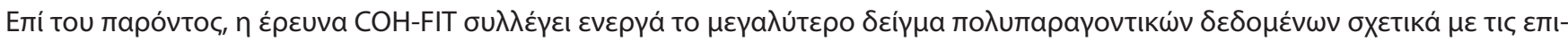

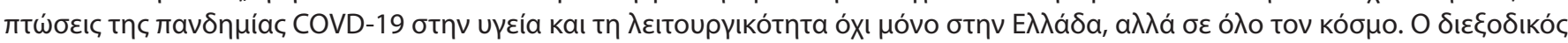

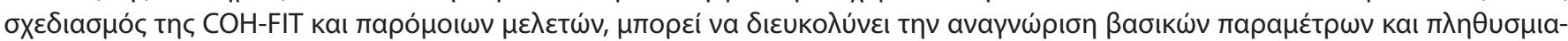

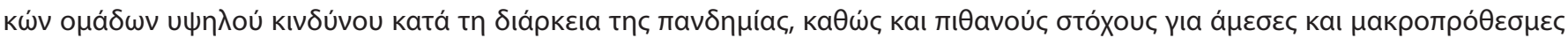

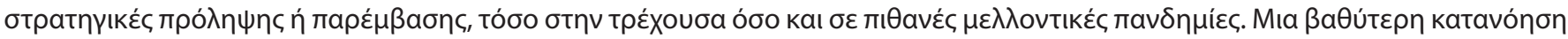

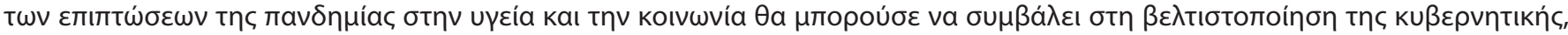

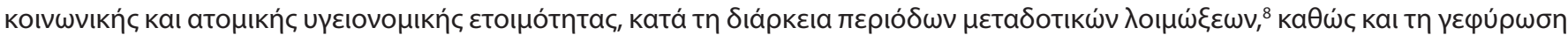

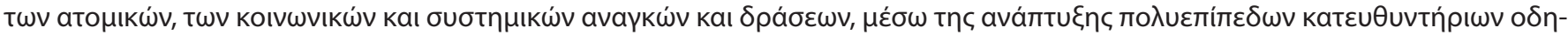

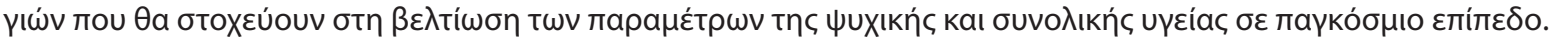

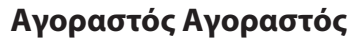

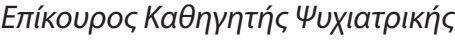

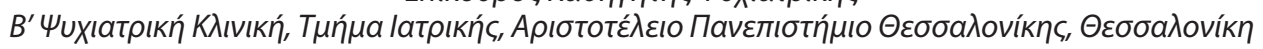

Kwvotavtivoc Toauákns

Research Associate

Institute of Psychiatry, Psychology and Neuroscience, King's College London, UK.

Marco Solmi

Assistant Professor of Psychiatry

Department of Neuroscience, University of Padua, Italy

Christoph U. Correll

Professor of Child and Adolescent Psychiatry

Department of Child \& Adolescent Psychiatry, Psychotherapy and Psychosomatics,

Charité University Medical Center Berlin, Germany

Professor of Psychiatry and Molecular Medicine

Donald and Barbara Zucker School of Medicine at Hofstra/Northwell Hempstead, NY, USA

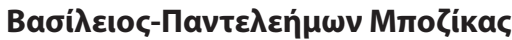

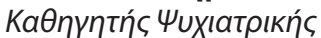

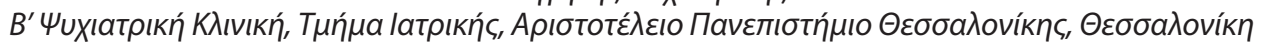

\section{References}

1. COVID-19 Dashboard Center for Systems Science and Engineering, Johns Hopkins University. Available from: https://www.arcgis.com/apps/opsdashboard/index.html\#/bda7594740fd40299423467b48e9ecf6. Accessed 31.12.2020

2. Horton R. Offline: COVID-19 is not a pandemic. Lancet 2020, 396:874, doi: 10.1016/S0140-6736(20)32000-6

3. Taylor S, Landry CA, Paluszek MM, Fergus TA, McKay D, Asmundson GJG. COVID stress syndrome: Concept, structure, and correlates. Depress Anxiety 2020, 37:706-714, doi: 10.1002/da.23071

4. Luo Y, Chua CR, Xiong Z, Ho RC, Ho CSH. A Systematic Review of the Impact of Viral Respiratory Epidemics on Mental Health: An Implication on the Coronavirus Disease 2019 Pandemic. Front Psychiatry 2020, 11:565098, doi: 10.3389/fpsyt.2020.565098
5. Salazar de Pablo G, Vaquerizo-Serrano J, Catalan A, Arango C, Moreno C, Ferre F et al. Impact of coronavirus syndromes on physical and mental health of health care workers: Systematic review and meta-analysis. J Affect Disord 2020, 275:48-57, doi: 10.1016/j.jad. 2020.06.022

6. Kuntz JC. Resilience in times of global pandemic: Steering recovery and thriving trajectories. Appl Psychol 2020, Online ahead of print, doi: 10.1111/apps.12296

7. Tsamakis K, Dimitrakakis G, Stefanadi E, Tsiptsios DA, Dimitrakaki I, Mueller $\mathrm{C}$ et al (Comment) The challenges of planetary mental health in the COVID-19 era. Exp Ther Med 2020, 20:1843-1844, doi: 10.3892/etm. 2020.8927

8. Ahmad A, Mueller C, Tsamakis K. Covid-19 pandemic: a public and global mental health opportunity for social transformation? BMJ 2020 369:1383, doi: 10.1136/bmj.m1383 